\title{
Automatic Selection Tool of Quality Control Specifications for Off- site Construction Manufacturing Products: A BIM-based Ontology Model Approach
}

\author{
Pablo Martinez ${ }^{1}$, Rafiq Ahmad ${ }^{2 *}$, and Mohamed Al-Hussein ${ }^{3}$ \\ ${ }^{1}$ PhD Student, Department of Civil Engineering, University of Alberta \\ ${ }^{2}$ Assistant Professor, Department of Mechanical Engineering, University of Alberta \\ ${ }^{3}$ Professor, Department of Civil Engineering, University of Alberta \\ "Corresponding author's e-mail: rafiq.ahmad@ualberta.ca
}

\begin{abstract}
Construction manufacturing specifications play an important role in assessing quality requirements on a construction project. However, working with these specifications can be overly complicated and error prone to the large amount of regulations and codes that need to be considered and their inter-dependencies. In building information modelling (BIM), the model is a digital representation of a complex construction product and contains precise product information data. The data is currently embedded into the model as properties for parametric building objects that are exchangeable among project operators. Some effort has been previously done to enhance the BIM model to obtain construction-oriented data and linking information that is crucial to manufacturing and quality control and assurance with BIM modelling still remains a challenge. This study proposes an extension to the current BIM-based product-oriented ontology model to include manufacturing processes and inspection, and quality control specifications. By automatically identifying which specifications are applicable to certain products and to extract the requirements imposed, this approach can support and enable automatic decision making in quality inspection and control tasks, which solely depend on information and knowledge from construction specifications. This approach is tested and validated using a light-gauge steel frame wall under Canadian construction standards and regulations.
\end{abstract}

\section{KEYWORDS}

Quality control specifications; Off-site construction manufacturing; BIM; Ontology modelling; Modular construction.

\section{INTRODUCTION}

In any construction process, quality control and early defect detection are still the most important approaches to reduce project schedule delays and cost overruns. In modular construction, as automation and robotics are being integrated, real-time quality control is becoming a key factor in the manufacturing process. Presently, online product inspection is a necessary requirement of any automatic or semi-automatic manufacturing process (Di Leo et al. (2017)). Current approaches for quality control and assurance in off-site construction facilities are mostly paperbased, time-consuming, and ineffective since data is only provided at specific locations and times. Effectively identifying potential defects early in the manufacturing process is critical for planning and productivity. Patterson and Ledbetter (1989) reported that 6 to 12\% of construction 
cost is wasted because of rework of defective components detected during the construction phase, and an additional 5\% was detected during maintenance procedures later on. Most of those defects were attributed to errors during the manufacturing phase.

Despite technological advancements in information and management in the construction industry, a link between quality control and product manufacturing information models is still missing. Initially, to address the aforementioned problems, Akinci et al. (2006) defined the formalism needed to utilize sensor systems for active quality control for general construction sites, considering inspection goals, planning, data collection, defect detection, and management. The proposed model was integrated into the management and design modelling of construction products comparing 3D scanned images with highly detailed 3D models. A few years later, Chen and Luo (2014) addressed the need to link the BIM to current quality control procedures. The proposed integrated solution offered a bridge between BIM environment models and quality management processes from a very generic perspective. The richness of design information offered by Building Information Models (BIM) has helped in the delivery of improved quality buildings. BIM itself is a purpose-built, product-centric information database. For off-site construction, the ability to extract construction manufacturing specific information from a BIM model is critical to support productive workplaces. An example of such implementation could be found in Wang et al. (2015) study, which defines the quality control of construction works onsite in two main steps: (1) LiDAR-based real-time tracking system, and (2) integrated BIM-based real-time checking system. This vision-based system can automatically collect data, perform comparisons between the as-built data and as-planned BIM model, and assess construction quality on-site. Automatic quality control on-site was targeted by Boukamp and Akinci (2007), to identify specifications applicable to products existing in a construction project and extract the requirements imposed by each product specification. Malik et al. (2019) successfully extracted manufacturing-related product specifications from BIM with the purpose of generating safe toolpaths. In terms of construction quality control and assessment, the current quality requirements and knowledge available are through mandated international, national or local standards, regulations and codes, and added company-specific directives, thus making construction quality related knowledge and project specific information scattered and fragmented. Martinez et al. (2019) designed a vision-based system, using manufacturing information from the BIM model, for real-time inspection of steel frame assemblies. The real-time comparison between BIM model and real frame components offers an extra dynamic layer of security regarding quality control and assurance in off-site construction.

The complex and dynamic nature of construction manufacturing and its off-site work patterns are widely known. The requirements imposed by construction specifications should be known for both planning for inspection and quality control. The integration of vision-based systems on BIM-based construction processes has facilitated a deeper understanding and control over the quality control of construction related manufactured products. However, most of the current vision-based systems designed for the construction industry are product-specific or task-specific and cannot be utilized for general manufacturing purposes. To address such shortcomings, ontology models have proven effective in the construction environment. Gruber (1995) defined ontology as "an explicit and formal specification of a conceptualization". In recent literature, ontology models have proven successful in creating knowledge links between construction related domains. The proposal named MASON (MAnufacturing's Semantics ONtology) proposed by Lemaignan et al. (2006) created a common semantic net in the manufacturing 
environment using ontologies for general purposes. This approach successfully links related product specifications and manufacturing related resources. MASON sets the foundation to link construction-oriented products to its manufacturing environment. Zhang et al. (2015) proposed an ontology-based model to link on-site construction safety planning with current job hazards. Their system automatically provides site managers with a list of equipment, material, labour, and safety measures by linking construction tasks and methods, and their potential hazards, with industry safety experts' knowledge and safety specifications. Lee et al. (2014) defined a semantic ontology model to accurately estimate the total cost, considering materials, labour and equipment, of tiling in a building construction project based on BIM. Recently, Liu et al. (2016) defined an ontology-based semantic approach to obtain quantity take-off information from BIM models for light-frame buildings. As such, construction practitioners can obtain the relevant construction-oriented information easily from complex 3D building models.

Following a similar approach, this paper proposes an extension of the ontology model created by Liu et al. (2016) to include the integration of vision-based systems for quality control of building components that can be manufactured in off-site construction facilities by linking the manufactured product and its production line with manufacturing operations, as proposed by Lemaignan et al. (2006), company specifications, and manufacturing standards.

\section{METHODOLOGY}

The purpose of developing a construction quality control ontology is not only to formalize the current construction quality control and inspection knowledge, but also to support defect identification during off-site manufacturing processes and mitigation through BIM. An ontology model should then support integration of the knowledge from building information modeling. Following the pre-existing architecture, an ontology-augmented BIM-based ontology model is developed to automatically identify regulations and standards, as well as company specifications if they were to exist, that are linked to the desired construction product. The ease of access to such knowledge would support quality control managers in advancing decision making for product inspection planning along its production line, focusing on the key features of the product at each stage of the manufacturing process.

In order to make off-site construction-related manufactured products' quality control inspections easier and more efficient, an ontology modelling of quality control specifications is explored. The overall detailed architecture of the proposed system is shown in Figure 1, extended to quality control (light blue databases) from previous work by Liu et al. (2016) on constructionoriented product ontology formulation (grey database and programs) and previous work by Lemaignan et al. (2006) on manufacturing operations (light green program). The developed ontology model (see Figure 2) consists of three main modules that represent the product (Construction-oriented Product Ontology), the associated manufacturing process (Manufacturing-oriented Product Ontology), and the quality control specifications proposed for such product (Quality Control Ontology). 


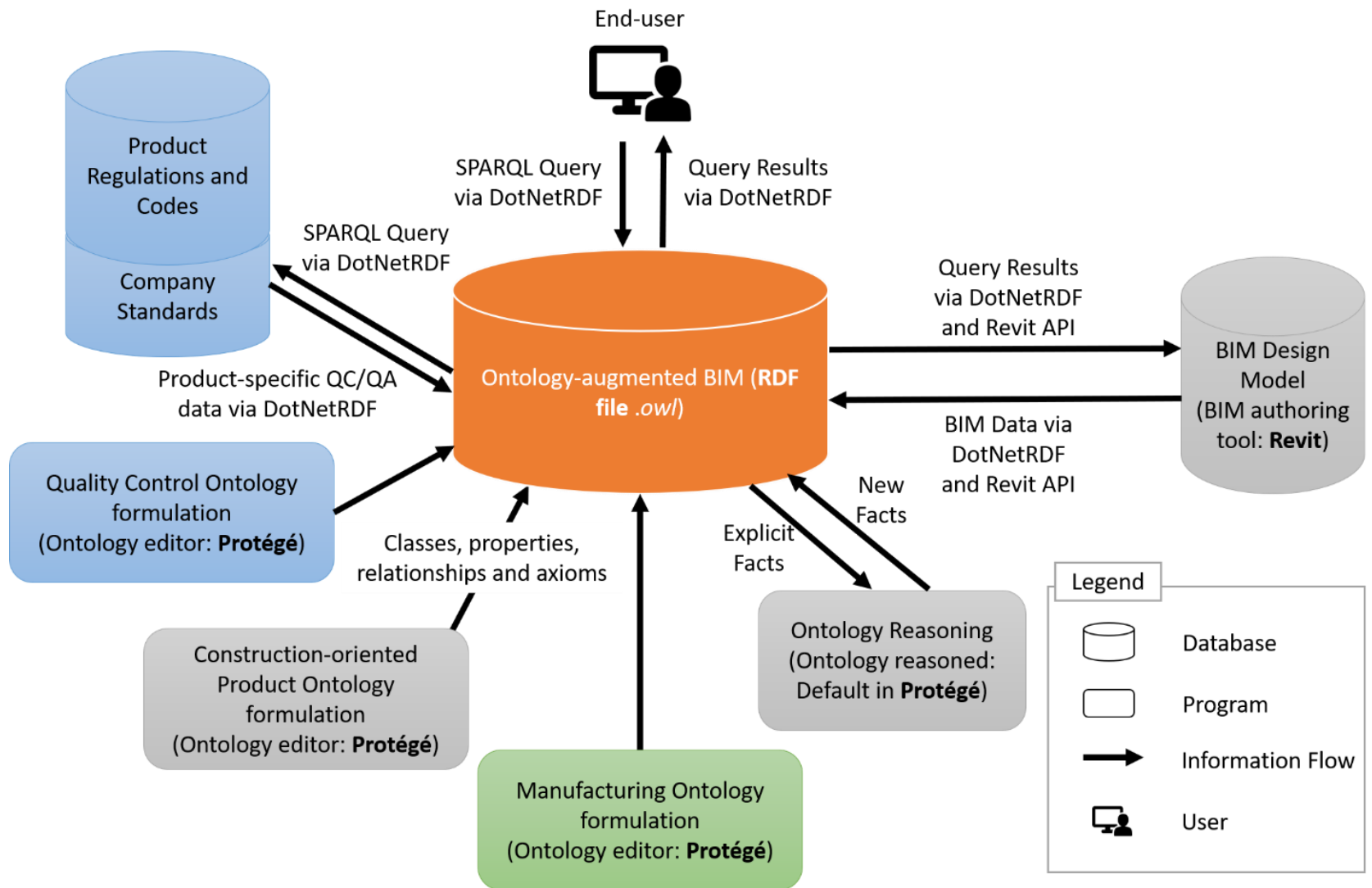

Figure 1. Proposed system architecture.

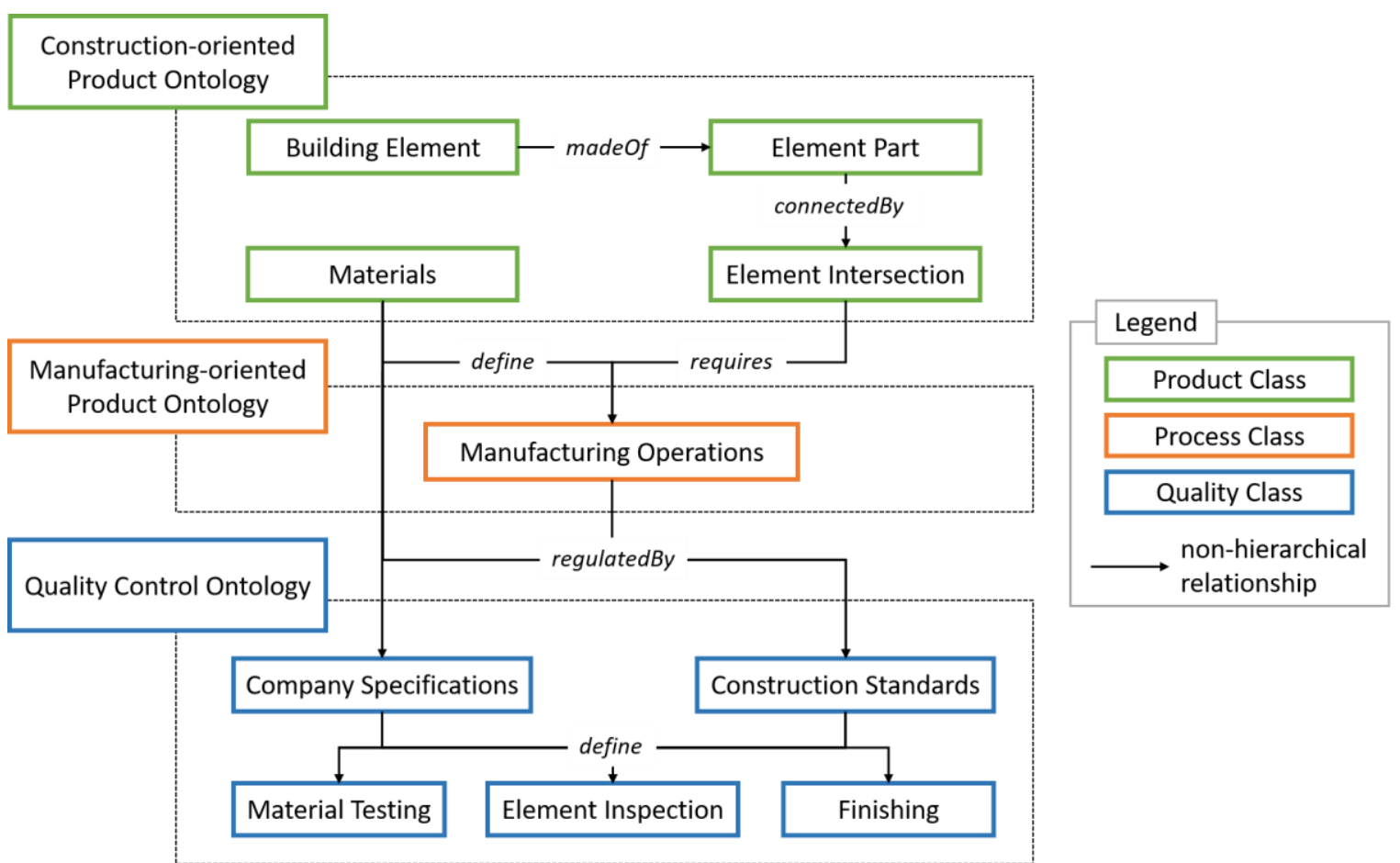

Figure 2. Ontology model for quality control in construction manufactured products. 
The Construction-oriented Product Ontology contains building element information, such as studs, windows, and joists, and provides the main interface for connecting the ontology with a BIM platform. BIMs are digital representations of physical and functional characteristics of a facility and contain all the information for each component of a product. This ontology model was taken from work by Liu et al. (2016), although some modifications were done to accommodate the manufacturing and quality control extension. In terms of construction of a building element, the following information will be used to allocate manufacturing resources: material, dimension, and intersection between elements. An intersection is defined as the interface between any two or more members to be connected. Since an intersection is dominantly affected by the product material, layer location, and geometric shape, each intersection is specific to each pair (or more) of products.

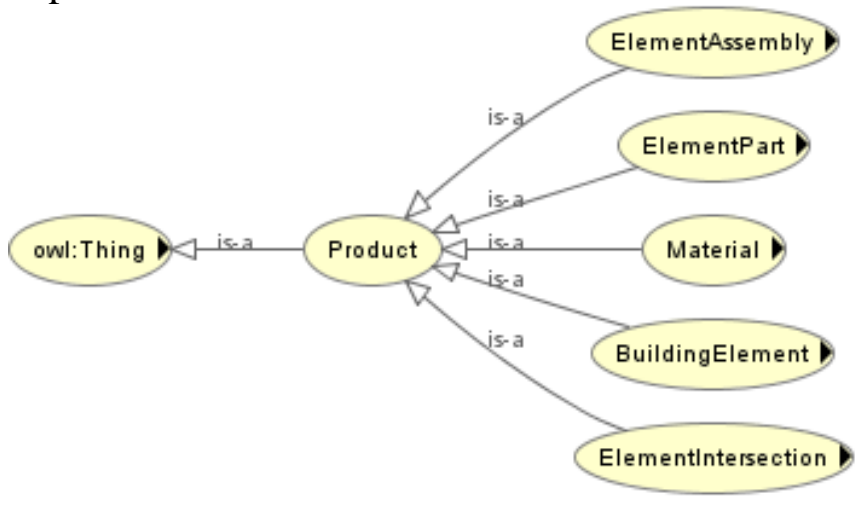

Figure 3. Proposed construction product ontology model.

The Manufacturing-oriented Product Ontology includes the manufacturing operations required for the construction product. This model was leveraged from the model proposed by Lemaignan et al. (2006) and modified to fit the off-site construction manufacturing domain. 'Manufacturing Operations' is related to the BIM 'Element Intersection' through the 'requires' property, i.e., Stud_Stud_PlainSurface requires Screw Fastening, Nailing or Welding (among other possibilities). The manufacturing operation may consist of a set of construction methods. The construction method is also defined by the materials used during the activity: the hard connection between studs in construction differs if the stud's material is wood or steel.

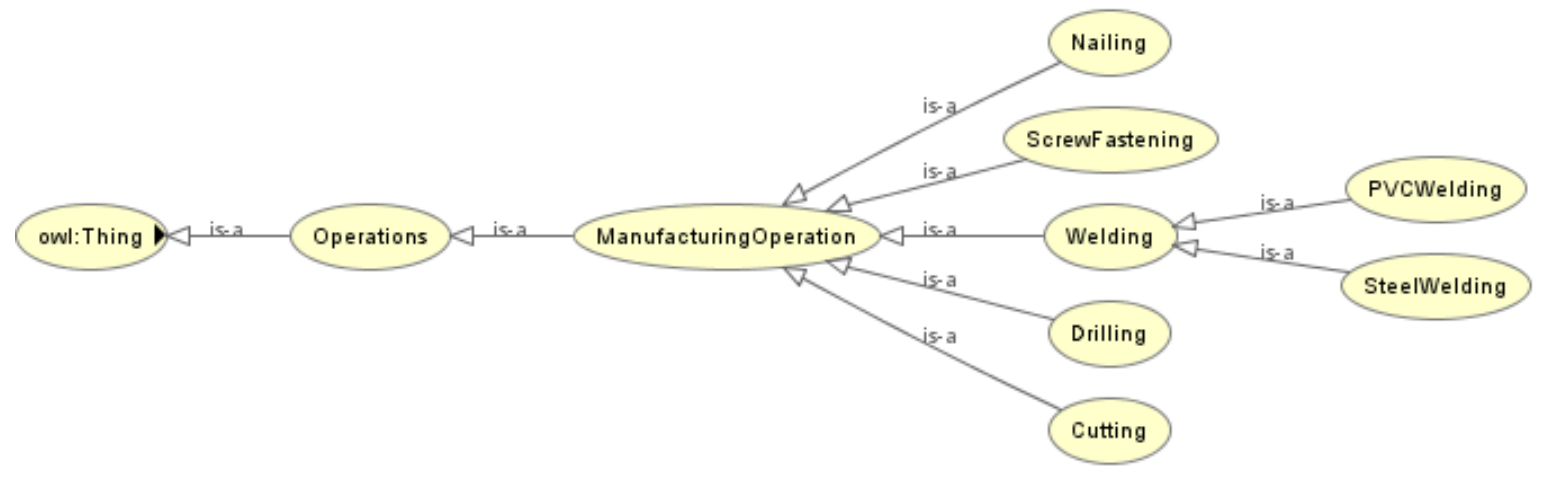

Figure 4. Proposed manufacturing operations ontology model. 
The Quality Control Ontology links the construction quality control and assurance information from international, national, local standards, regulations or codes, as well as product-specific company specifications with the manufacturing and product information mentioned previously. Such regulations are stored as a database and are linked to a 'Material' and/or 'Manufacturing Operation' and define the quality control that is necessary for the manufactured product. For construction manufactured products, three different areas for quality control and assurance are defined: (1) 'Material Testing' is the requirement to test the physical properties of the materials used on the product; (2) 'Element Inspection' is the inspection of the product as a whole, focusing on defects that may endanger the integrity of the product (geometry, missing elements, wrong materials, etc.); (3) 'Finishing' relates to the aesthetic inspection of the product, which is in most cases specific to the company.

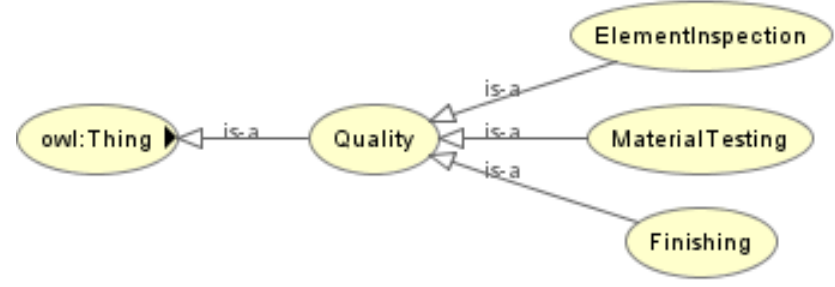

Figure 5. Proposed quality control ontology model.

\section{CASE STUDY AND RESULTS}

The proposed methodology is validated with a light-gauge steel (LGS) wall, designed in Revit (BIM software), as shown in Figure 6. In order to populate the ontology model with BIM data, the ontology model is saved into an RDF file (owl extension). DotNetRDF provides functions such as RDFGraphAssert to generate individuals within the ontology model with BIM data.

Note that the intersections between different elements are separated into two different groups: intersections generated within the same wall layer, intra-layer intersections, and intersections necessary to link the different layers within the wall, interlayer intersections. For this panel, all types of intersections are identified and matched to the ontology model as individuals. These intersections are annotated and tabulated as shown below.

Table 1. Summary of intersection types defined in the LGS panel with their corresponding possible manufacturing operations.

\begin{tabular}{|c|c|c|c|}
\hline & Intersection Type & Identifier & $\begin{array}{c}\text { Manufacturing } \\
\text { Operations }\end{array}$ \\
\hline Intra-layer & Stud_Stud_PlainSurface_LConnection & SS_PS_L & $\begin{array}{l}\text { ScrewFastening, } \\
\text { Welding }\end{array}$ \\
\hline \multirow{4}{*}{ Interlayer } & Sheathing_Frame_Connection & SH_FR & $\begin{array}{c}\text { ScrewFastening, } \\
\text { MagneticAttachments }\end{array}$ \\
\hline & Drywall_Frame_Connection & DW_FR & ScrewFastening \\
\hline & HouseWrap_Sheathing_Connection & HW_SH & $\begin{array}{l}\text { CapFastening, } \\
\text { CapStapling }\end{array}$ \\
\hline & Cladding_HouseWrap_Connection & CL_HW & ScrewFastening \\
\hline
\end{tabular}



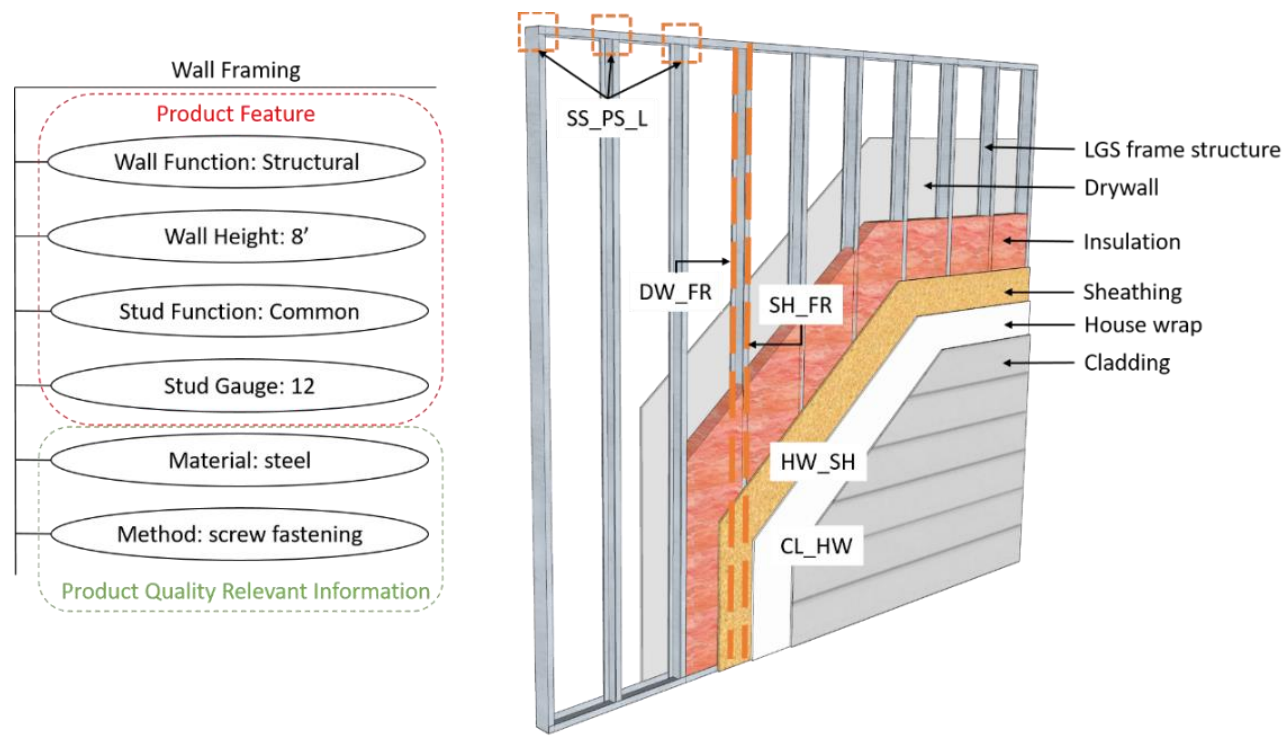

Figure 6. Graphical representation of the light-gauge steel wall panel layers and intersection types.

Finally, to simulate the entry of data from a user perspective, a semantic SPARQL query to search for the quality control specifications related to this construction product is made. The query is executed through the dotNetRDF that is adopted and integrated within the Revit add-on prototyped by Liu et al. (2016) The query itself and the results are shown in the table below.

Table 2. Semantic SPARQL query result to check inspection quality specifications for the LGS frame given.

SELECT Distinct (str(?QualitySpec) as ?Specification)

WHERE \{ ?hostWall rdf:type proOnto:StudFramedWall

Query ?hostWall proOnto:Material ?material . ?hostWall proOnto:mOperation ?moperation . FILTER (?Specification $=$ "ElementInspection"^^x ${ }^{\mathrm{X}} \mathrm{xd}$ :string) . FILTER (?Range $=$ "Canada" ${ }^{\wedge \wedge}$ Xsd:string). \}

National Building Code of Canada

CSA S136 North American Specification for the Design of Cold-Formed Steel Structural Members

Results AISI North American Standard for Cold-Formed Steel Framing - Product Data ASTM C1007-11a (2015) Standard Specification for Installation of Load Bearing Steel Studs ASTM C645 Standard Specification for Nonstructural Steel Framing Members

\section{CONCLUSIONS}

To select the appropriate construction specifications for a designed BIM-based product can be a challenging task, especially when dealing with newer products. This paper proposes an ontologybased approach to integrate, within the BIM environment, manufacturing operations, and quality control and assurance specifications. This approach enables quality operators to automatically query which quality specifications need to be considered during the manufacturing process. Using simple SPARQL queries, a list of all the quality specifications required is given. 
As the current system only uses partial information from the BIM model, the semantic queries that must be inputted to the system to generate the list of quality control and assurance specifications are quite simple and predictable. Future work will aim to automatically generate those queries within the BIM environment for ease of use. Further, the ontology model will be extended to other off-site construction facilities, other than steel panel manufacturing, such as wood panel manufacturing, window manufacturing, or door manufacturing among others.

\section{REFERENCES}

Patterson, L. \& Ledbetter, W. (1989). "The cost of quality: a management tool, excellence in the constructed project". Proceedings of Construction Congress I, ASCE.

Akinci, B., Boukamp, F., Gordon, C., Huber, D., Lyons, C. \& Park, K. (2006). "A formalism for utilization of sensor systems and integrated project models for active construction quality control". Automation in Construction, 15(2), 124-138.

Boukamp, F. and Akinci, B. (2007). "Automated processing of construction specifications to support inspection and quality control". Automation in Construction, 17, 90-106.

Chen, L. \& Luo, H. (2014). "A BIM-based construction quality management model and its applications". Automation in Construction, 46, 64-73.

Lemaignan, S., Siadat, A., Dantan, J.-Y. and Semenenko, A. (2006). "MASON: A Proposal for an Ontology of Manufacturing Domain," in Proceedings of the IEEE Workshop on Distributed Intelligent Systems: Collective Intelligence and Its Applications.

Wang, J., Sun, W., Shou, W., Wang, X., Wu, C., Chong, H.Y., Liu, Y. \& Sun, C. (2015). "Integrating BIM and LiDAR for Real-Time Construction Quality Control". Journal of Intelligent and Robotic Systems, 79, 417-432.

Malik, N., Ahmad, R. \& Al-Hussein, M. (2019). "Generation of safe tool-paths for automatic manufacturing of light gauge steel panels in residential construction". Automation in Construction, 98, 46-60.

Martinez, P., Ahmad, R. \& Al-Hussein, M. (2019). "A vision-based system for pre-inspection of steel frame manufacturing”. Automation in Construction, 97, 151-163.

Gruber, T.R. (1995). "Toward principles for the design of ontologies used for knowledge sharing". International Journal of Human-Computer Studies, 43(5), 907-928.

Zhang, S., Boukamp, F. \& Teizer, J. (2015). "Ontology-based semantic modelling of construction safety knowledge: Towards automated safety planning for job hazard analysis (JHA)". Automation in Construction, 52, 29-41.

Liu, H., Lu, M. \& Al-Hussein, M. (2016). "Ontology-based semantic approach for constructionoriented quantity take-off from BIM models in the light-frame building industry". Advanced Engineering Informatics, 30, 190-207.

Lee, S.-K., Kim, K.-R. \& Yu, J.-H. (2014). "BIM and ontology-based approach for building cost estimation". Automation in Construction, 41, 96-105.

Benevolenskiy, A., Roos, K., Katranuschkov, P. \& Scherer, R.J. (2012). “Construction processes configuration using process patterns". Advanced Engineering Informatics, 26 (4), 727-736.

Wang, H.-H. \& Boukamp, F. (2011). "Ontology-based representation and reasoning framework for supporting job hazard analysis". ASCE Journal of Computing in Civil Engineering, 25(6), 442-456.

Di Leo, G., Liguori, C., Pietrosanto A. \& Sommella, P. (2017). A vision system for the online quality monitoring of industrial manufacturing, Optics and Lasers in Engineering, 89, 162168. 